\title{
Manipulation of ferromagnets via the spin-selective optical Stark effect
}

\author{
Alireza Qaiumzadeh, ${ }^{1}$ Gerrit E. W. Bauer, ${ }^{2,3,4}$ and Arne Brataas ${ }^{1}$ \\ ${ }^{1}$ Department of Physics, Norwegian University of Science and Technology, NO-7491 Trondheim, Norway \\ ${ }^{2}$ Institute for Materials Research, Tohoku University, Sendai 980-8577, Japan \\ ${ }^{3}$ WPI Advanced Institute for Materials Research, Tohoku University, Sendai 980-8577, Japan \\ ${ }^{4}$ Delft University of Technology, Kavli Institute of NanoScience, 2628 CJ Delft, The Netherlands \\ (Received 15 January 2013; revised manuscript received 9 August 2013; published 19 August 2013)
}

\begin{abstract}
We investigate the nonresonant all-optical switching of magnetization. We treat the inverse Faraday effect (IFE) theoretically in terms of the spin-selective optical Stark effect for linearly or circularly polarized light. In the dilute magnetic semiconductors $(\mathrm{Ga}, \mathrm{Mn}) \mathrm{As}$, strong laser pulses below the band gap induce effective magnetic fields of several teslas in a direction which depends on the magnetization direction as well as the light polarization and direction. Our theory demonstrates that the polarized light catalyzes the angular momentum transfer between the lattice and the magnetization.
\end{abstract}

DOI: 10.1103/PhysRevB.88.064416

PACS number(s): 75.78.-n, 71.70.Ej, 75.40.Gb, 78.20.Bh

\section{INTRODUCTION}

An essential challenge in magnetoelectronics is finding new methods to manipulate the magnetization and increase the switching speed for realizing faster and higher-density data storage and information processing. Traditionally, the magnetization is switched by applying nearly collinear external magnetic fields for longer than 100 ps. ${ }^{1}$ Current-induced spin transfer torque switching is not faster. ${ }^{2}$ So-called precessional switching can be achieved by a magnetic field pulse perpendicular to the magnetization,,$^{1,3}$ leading to magnetization reversal as fast as, but not faster than, a few ps. ${ }^{3}$ In this paper, we explain how off-resonant optical pulses generate strong effective magnetic fields that can lead to ultrafast magnetic reversal.

Recently, it has been demonstrated that a 40 fs circularly polarized pulse can reverse the magnetization of the metallic ferrimagnet $\mathrm{GdFeCo}$ with perpendicular magnetic anisotropy. ${ }^{4}$ A magnetic write event as short as 30 ps by using $100 \mathrm{fs}$ circularly polarized light pulses has been reported, ${ }^{5}$ i.e., a potential data storage rate of about $10 \mathrm{Tbit} / \mathrm{s}$. Simulations suggest that the magnetization reversal is not realized via precession, but is caused by a linear process in which the magnitude of the magnetization passes through zero, in the presence of magnetic fields of $\sim 20 \mathrm{~T} .{ }^{4,5}$ The magnetization dynamics can also be triggered by light that is linearly polarized in a direction noncollinear to the crystal axis. ${ }^{6,7}$ For a comprehensive review on ultrafast all-optical magnetization dynamics, see Ref. 8. Phenomenologically, these experiments are attributed to two reactive effects: (i) the inverse Faraday effect (IFE), ${ }^{9,10}$ the ability of the electric field component of circularly polarized light $\mathbf{E}(\omega)$ to induce a static magnetization $\mathbf{M}_{\mathrm{IFE}}(0) \propto \mathbf{E}(\omega) \times \mathbf{E}^{*}(\omega)$; and (ii) the inverse Cotton-Mouton effect (ICME), ${ }^{10}$ the magnetization induced by polarized light in the presence of an external magnetic field $\mathbf{B}_{\text {ext }}, \mathbf{M}_{\mathrm{ICME}}(0) \propto$ $|\mathbf{E}(\omega)|^{2} \mathbf{B}_{\text {ext }}$.

The microscopic origin of the large magnetic field induced by light and the induced magnetization dynamics are still a subject of debate. ${ }^{8,11,12}$ Several theoretical mechanisms have been proposed, such as the optical Barnett effect or the inverse Einstein-de Haas effect, ${ }^{13}$ light-induced circular currents in the collisionless limit, ${ }^{14,15}$ the impulsive stimulated Ramanlike process, ${ }^{8}$ and photonic angular momentum transfer via deflection of the scattered photons. ${ }^{16}$ A dissipative IFE under $\mathrm{THz}$ irradiation has been computed for dirty metals with extrinsic spin-orbit interaction. ${ }^{17,18}$ Also experimentally the situation is not clear. Recent experiments on the ferrimagnetic metallic alloy $\mathrm{GdFeCo}$ found optical magnetization switching without light polarization in a certain range of light intensities and sample temperatures, casting doubt on the ubiquity of the IFE. ${ }^{19}$ Such a behavior was not reported for TbCo films, however. ${ }^{20}$

In this paper, we predict huge effective magnetic fields induced by the below-band-gap polarized light through the spin-selective ac (optical) Stark effect, ${ }^{21}$ i.e., the shift of electronic energy levels connected through finite optical matrix elements. ${ }^{10}$ In perturbation theory, this process is closely related to Raman scattering. Since the electronic structure of amorphous alloys is complicated and experiments concerning the role of the IFE are inconclusive, we focus here on GaMnAs as a generic model system, since it can be grown with perpendicular anisotropy and its electronic structure is well known. Dilute magnetic semiconductors are interesting spintronic materials by themselves. ${ }^{22}$ Although their Curie temperature at present is below room temperature, studying these materials can improve our understanding of novel physical phenomena that are also present in other magnets. ${ }^{23-25}$ Photoinjected carriers induced by linearly polarized light with frequency slightly above the $\Gamma$ or $L$ band edges have been shown to induce magnetization dynamics in GaMnAs. ${ }^{23,26}$ In contrast, we focus here on excitation with frequencies below the fundamental band gap, which is dissipationless, since no free carriers are excited. Our approach is quite general and can be applied to arbitrary electronic structures and computed from first principles.

In one scenario, ${ }^{4,5}$ extrinsic processes due to the highintensity laser pulse heat up the ferromagnet so that it becomes paramagnetic while the circularly polarized light generates the spin-selective optical Stark effect or effective magnetic field $B_{\mathrm{IFE}}$ that triggers linear reversal, i.e., the modulus of the magnetization passes through zero during switching. The maximum achievable field can be huge, e.g., 
for GaAs parameters $B_{\mathrm{IFE}}=\hbar \Omega /\left(g_{s}^{*} \mu_{B}^{*}\right) \sim 100 \mathrm{~T}$, where $\Omega$ is the Rabi frequency for a light intensity of $5 \mathrm{GW} \mathrm{cm}^{-2}$, and $g_{s}^{*} \mu_{B}^{*} / \hbar \sim 500 \mathrm{GHz} / \mathrm{T}$ is the effective gyromagnetic ratio. In practice, the light frequency should be sufficiently below the band gap to reduce heating that destroys the sample at high intensities. We therefore formulate the IFE here in second-order perturbation theory and compute the resulting expressions for the GaAs band structure and wave functions. We find that, for light frequencies safely below the energy gap, the effective field amounts to several teslas in GaMnAs, which suffices to nucleate a ferromagnetic state during the cooling phase. ${ }^{5,27}$ In a second scenario, we assume that the material remains ferromagnetic under the laser excitation, possibly with a reduced magnetization. In this case, both linearly and circularly polarized light can trigger both precessional and linear switching mechanisms. In both scenarios, the required angular momentum is not supplied by the photons, but by the lattice via the spin-orbit interaction. We show that, in general, the light-induced effective field has three components, depending on the light polarization and initial magnetization direction, viz., $\mathbf{B}_{\text {light }}=B_{\mathrm{IFE}} \hat{\mathbf{q}}+B_{\mathrm{ICME}} \hat{\mathbf{M}}_{0}+B_{\perp} \hat{\mathbf{q}} \times \hat{\mathbf{M}}_{0}$. The sign of the IFE effective field $B_{\text {IFE }}$ depends on the helicity and points along the light propagation direction $\hat{\mathbf{q}}$. $B_{\mathrm{ICME}}$, the magnetic field associated with the ICME, is directed along the magnetization vector $\hat{\mathbf{M}}_{0}$, while a field with strength $B_{\perp}$ is perpendicular to both.

This paper is organized as follows. In Sec. II we present the Kane band model we have used to describe light-matter interaction and introduce the effective Hamiltonian within the second-order time-dependent perturbation theory. In Sec. III we present and discuss our main analytical results for the lightinduced magnetic field in dilute magnetic semiconductors. Finally, in Sec. IV we present a summary of our main conclusions.

\section{MODEL HAMILTONIAN AND SECOND-ORDER TIME-DEPENDENT PERTURBATION THEORY}

In the Coulomb gauge, the eight-band Kane model Hamiltonian for a zinc-blende semiconductor at the $\Gamma$ point reads $\mathcal{H}=\mathcal{H}_{0}+\mathcal{H}_{p-d}+\mathcal{H}_{\text {int }}$, where

$$
\begin{aligned}
\mathcal{H}_{0} & =\frac{\mathbf{p}^{2}}{2 m}+\frac{\hbar}{4 m^{2} c^{2}} \mathbf{p} \cdot\left(s \times \nabla V_{p}\right)+V_{p}, \\
\mathcal{H}_{s p-d} & =-J \hat{\mathbf{M}_{0}} \cdot \boldsymbol{s}, \\
\mathcal{H}_{\text {int }} & \simeq \frac{e}{m c} \mathbf{A} \cdot \mathbf{p}+\frac{e^{2}}{2 m c^{2}} \mathbf{A}^{2} .
\end{aligned}
$$

Here $\boldsymbol{p}, e$, and $m$ are the momentum operator, electron charge, and electron mass, respectively, $\hbar$ is the reduced Planck constant, $c$ is the light velocity, $s$ is vector of $2 \times 2$ Pauli matrices, $\mathbf{A}$ is the vector potential of the monochromatic light field, $V_{p}$ is the periodic lattice potential, and $\mathcal{H}_{s p-d}$ is the $s p-d$ mean-field exchange interaction between the magnetization direction of the localized $d$ spins $\hat{\mathbf{M}}_{0}$, and the itinerant $s$ or $p$ spins, controlled by the exchange potential $J$. The $\mathbf{A} \cdot \mathbf{p}$ interaction term describes the annihilation of a photon and the creation of an electron-hole pair and vice versa, while $\mathbf{A}^{2}$ represents a photon scattering processes. In perturbation theory, two-photon transitions can be induced by either $\mathbf{A} \cdot \mathbf{p}$ to second order or $\mathbf{A}^{2}$ to first order in the interaction Hamiltonian. To leading order in the light-matter interaction, $\mathbf{A}^{2}$ does not induce spin reversal and will therefore be disregarded in the following. A · p induces only two-photon virtual interband transitions, since the light frequency is below the band gap. Intraband transitions are disregarded because they are impurity mediated and weak. Direct-band-gap semiconductors can be treated in the effective mass approximation and projected on the well established eight-band Kane model for $\mathcal{H}$ including the conduction $\left(\left|\mathrm{cb}^{ \pm}\right\rangle\right)$, the heavy-hole $\left(\left|\mathrm{hh}^{ \pm}\right\rangle\right)$, the light-hole $\left(\left|\mathrm{lh}^{ \pm}\right\rangle\right)$, and the spin-orbit split-off $\left(\left|\mathrm{so}^{ \pm}\right\rangle\right)$bands. ${ }^{28}$ In the following, we will disregard the band dispersion, a common approximation in theories of Raman scattering ${ }^{29}$ that is allowed for low doping levels and/or large detuning.

The electric field component of monochromatic light with frequency $\omega_{0}$ and wave vector $\mathbf{q} \| \hat{\mathbf{z}}$ is $\mathbf{E}(t)=\hat{\mathbf{e}} E_{0} e^{-i\left(\omega_{0} t-\mathbf{q} \cdot \mathbf{r}\right)}+$ c.c., for light propagating along the $z$ direction with polarization $\hat{\mathbf{e}}=e_{x} \hat{\mathbf{x}}+e_{y} \hat{\mathbf{y}}$. For circular polarization $e_{x}=1 / \sqrt{2}$ and $e_{y}=\lambda i / \sqrt{2}$, where $\lambda= \pm 1$, and, for linear polarization with angle $\alpha$ relative to the $x$-axis, $e_{x}=\cos \alpha$ and $e_{y}=$ $\sin \alpha$. When the pulse duration $T_{p}$ is sufficiently longer than $\left(E_{g} / \hbar-\omega_{0}\right)^{-1}$, where $E_{g}$ is the energy gap, transient effects can be disregarded. ${ }^{30}$ For a laser pulse width of 40-100 fs with a frequency that is not too close to the resonance, the above criterion is satisfied by $\hbar / E_{g} \approx 0.5$ fs for our material.

The matrix elements of the Hamiltonian in second-order perturbation for the $\mathbf{A} \cdot \mathbf{p}$ interaction term $\operatorname{read}^{9}$

$$
\begin{aligned}
\langle m|\mathcal{H}| k\rangle= & \frac{e^{2} E_{0}^{2}}{m^{2} \omega_{0}^{2} c^{2}} \sum_{l}\left[\frac{\left\langle m\left|p_{\beta} e_{\beta}^{*}\right| l\right\rangle\left\langle l\left|p_{\gamma} e_{\gamma}\right| k\right\rangle}{\hbar \omega_{0}+\left(\epsilon_{k}-\epsilon_{l}\right)}\right. \\
& \left.-\frac{\left\langle m\left|p_{\gamma} e_{\gamma}\right| l\right\rangle\left\langle l\left|p_{\beta} e_{\beta}^{*}\right| k\right\rangle}{\hbar \omega_{0}-\left(\epsilon_{m}-\epsilon_{l}\right)}\right],
\end{aligned}
$$

where $\beta(\gamma)=x, y, z .|m\rangle,|k\rangle$, and $|l\rangle$ are the initial, final, and intermediate states including the spin and momentum quantum numbers, with the energies $\epsilon_{m}, \epsilon_{k}$, and $\epsilon_{l}$ respectively.

\section{LIGHT-INDUCED EFFECTIVE MAGNETIC FIELD IN GaAs AND GaMnAs}

Unlike an external magnetic field, conduction and valence bands experience different light-induced effective fields. ${ }^{31}$ For conduction band (valence bands) $\mathbf{B}_{\text {light }}=$ $2 \operatorname{Tr}[\mathbf{s} \mathcal{H}] /\left(\mu_{B}^{*} g_{s}^{*} \operatorname{Tr}\left[\mathbf{s}^{2}\right] / 3\right)$, where $\mathbf{s}$ is the vector of $2 \times 2$ Pauli spin matrices for $1 / 2$ spins in the conduction band (the $4 \times 4$ spin matrices for $3 / 2$ spins in the valence band), $\operatorname{Tr}$ is the trace over electron states (hole states), $\mu_{B}^{*}$ is effective Bohr magneton of the electron (hole), and $g_{s}^{*}$ is the electron (hole) effective Landé $g$ factor. Note that we lump heavy and light holes together by the trace and adopt an average value of $\mu_{B}^{*} g_{s}^{*}$ for the valence band.

As illustrated by Fig. 1, the below-band-gap light field induces a Zeeman-like splitting, called the spin-selective optical Stark shift, ${ }^{10}$ which can be interpreted as an effective magnetic field experienced by each band, $\delta E_{\text {Stark }}=-\mu_{B}^{*} g_{s}^{*} B_{\mathrm{IFE}} / 2$. The effective field, $B_{\mathrm{IFE}}$, is a reactive response and is therefore essentially instantaneous as long as $T_{p}>\hbar / E_{g}$. The effective field eventually gives rise to a nonequilibrium spinpolarization in the conduction band, $\left\langle\sigma_{z}\right\rangle_{\mathrm{ne}}^{(n)} \propto N^{(n)} \delta E_{\text {Stark }}$, where $N^{(n)}$ is the density of states per unit volume at the 


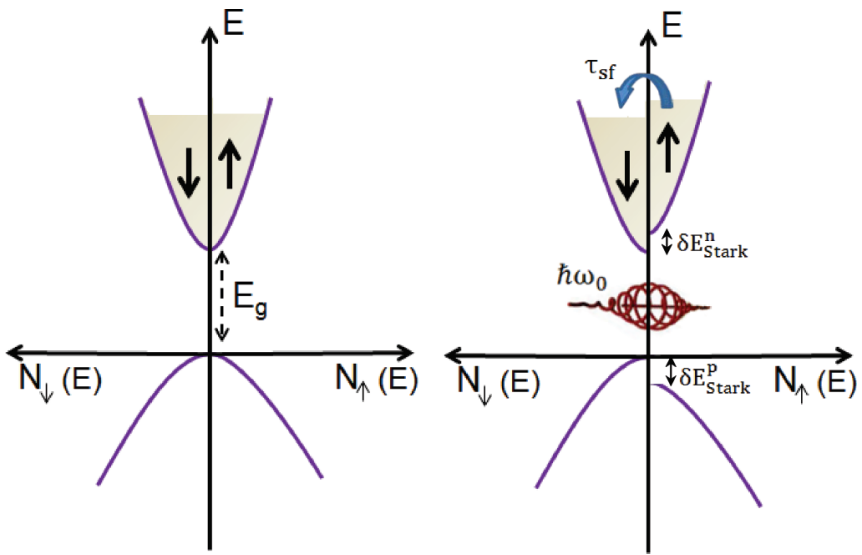

FIG. 1. (Color online) Illustration of the changes in the majority and minority population due to the Stark shift $\left(\delta E_{\text {Stark }}^{\mathrm{n}}<\delta E_{\text {Stark }}^{\mathrm{p}}\right)$, in the presence of a nonresonant and intense circularly polarized laser field.

Fermi level of the conduction band (with analogous relations for the holes) and $\langle\cdots\rangle$ denotes the expectation value on intermediate time scales. This spin polarization is generated by a repopulation of the states (see Fig. 1), on the scale of the spin-flip scattering time $T_{p} \sim \tau_{s f}$, which is expected to be in the ps range under high excitation conditions.

\section{A. Paramagnetic case}

In second-order time-dependent perturbation theory, the spin susceptibility of a paramagnet is defined as $\mathbf{K}_{\mathrm{IFE}}=$ $\langle\boldsymbol{\sigma}\rangle_{\mathrm{ne}} /\left(\xi E_{0}^{2} / \omega_{0}^{2}\right)$ with $\xi=e^{2} /\left(m c^{2}\right)$, which reads

$$
\begin{aligned}
\mathbf{K}_{\mathrm{IFE}}= & -\frac{1}{L^{3}} \sum_{k m} \frac{f\left(\epsilon_{k}\right)-f\left(\epsilon_{m}\right)}{\left(\epsilon_{k}-\epsilon_{m}\right)+i \eta}\langle k|\boldsymbol{s}| m\rangle \\
& \times\left[C_{\rho} \hat{\mathbf{e}} \cdot \hat{\mathbf{I}} \cdot \hat{\mathbf{e}}^{*}\langle m \mid k\rangle+i C_{\sigma}\left(\hat{\mathbf{e}} \times \hat{\mathbf{e}}^{*}\right) \cdot\langle m|\boldsymbol{s}| k\rangle\right],
\end{aligned}
$$

where $L$ is the system size, $\eta$ is a positive infinitesimal, $f(\epsilon)$ is the Fermi-Dirac distribution function in equilibrium, and $\hat{\mathbf{I}}$ is the unit dyadic in Pauli spin space. The interband couplings $C_{\sigma}$ and $C_{\rho}$ for $n$-doped semiconductors are

$$
\begin{aligned}
& C_{\sigma}^{(n)}=\frac{2 P^{2}}{3 m}\left(\frac{-\hbar \omega_{0}}{E_{g}^{2}-\hbar^{2} \omega_{0}^{2}}+\frac{\hbar \omega_{0}}{\left(E_{g}+\Delta\right)^{2}-\hbar^{2} \omega_{0}^{2}}\right), \\
& C_{\rho}^{(n)}=\frac{2 P^{2}}{3 m}\left(\frac{2 E_{g}}{E_{g}^{2}-\hbar^{2} \omega_{0}^{2}}+\frac{E_{g}+\Delta}{\left(E_{g}+\Delta\right)^{2}-\hbar^{2} \omega_{0}^{2}}\right),
\end{aligned}
$$

where $\Delta$ is the spin-orbit splitting energy and $P$ the interband momentum matrix element. The coefficients of $C_{\sigma}$ and $C_{\rho}$ are identical to the spin- and charge-density excitation coefficients in the theory of Raman scattering. ${ }^{29}$ In our formulation, the incoming and outgoing photons have identical polarization, Eq. (3), which means that there is no direct angular momentum transfer from the light to the medium. Angular momentum of spin-flip processes is hence supplied from the lattice via spin-orbit coupling during the spin-flip relaxation process.

In a paramagnetic $n$-doped semiconductor, the Stark effective field, or IFE field, is oriented along the light propagation direction $\hat{\mathbf{q}}$ as

$$
\mathbf{B}_{\mathrm{IFE}}^{(n)}=\frac{-2 \xi E_{0}^{2}}{\mu_{B}^{*} g_{s}^{*} \omega_{0}^{2}} \frac{\mathbf{K}_{\mathrm{IFE}}}{N^{(n)}}=-\frac{2 \lambda C_{\sigma}^{(n)} \xi E_{0}^{2}}{\mu_{B}^{*} g_{s}^{*} \omega_{0}^{2}} \hat{\mathbf{q}} .
$$

For $T_{p} \gtrsim \tau_{s f}$ this magnetic field leads to the spin accumulation $\langle\boldsymbol{\sigma}\rangle_{\text {ne }}^{(n)}=-K_{z, \mathrm{IFE}} \xi E_{0}^{2} \hat{\mathbf{q}} / \omega_{0}^{2}=-2 \lambda N^{(n)} C_{\sigma}^{(n)} \xi E_{0}^{2} \hat{\mathbf{q}} / \omega_{0}^{2}$.

In paramagnetic $p$-doped systems, the effective field experienced by the hole bands is

$$
\mathbf{B}_{\mathrm{IFE}}^{(p)}=-\frac{3 \lambda C_{\sigma}^{(p)} \xi E_{0}^{2} \hat{\mathbf{q}}}{\mu_{B}^{*} g_{s}^{*} \omega_{0}^{2}},
$$

where $C_{\sigma, \rho}^{(p)}=C_{\sigma, \rho}^{(n)}(\Delta \rightarrow \infty)$ since the matrix elements with the spin-orbit split-off bands vanish. In the $p$-doped case, the nonequilibrium spin polarization on longer time scales is $\langle\boldsymbol{\sigma}\rangle_{\text {ne }}^{(p)}=\lambda N^{(p)} C_{\sigma}^{(p)} \xi E_{0}^{2} \hat{\mathbf{q}} / \omega_{0}^{2}$, where $N^{(p)}$ is the average density of states at the Fermi level of hole bands.

According to Eqs. (6) and (7), polarized light with frequency $\omega_{0}<E_{g} / \hbar$ induces a magnetic field along $\hat{\mathbf{q}}$. Its sign is governed by the light helicity $\lambda$, while its magnitude is proportional to the light intensity $E_{0}^{2}$ and vanishes with the spinorbit coupling since $C_{\sigma}^{(n)}(\Delta=0)=0$. In $n$-doped systems and in the large-detuning limit $\omega_{0} \ll E_{g} / \hbar$, to leading order, it yields $\left\langle\sigma^{z}\right\rangle_{\text {ne }} \propto \Delta / \omega_{0}$ for $\Delta<E_{g}$. This optical Stark shiftinduced nonequilibrium spin polarization can be compared with the magnetization induced by the circular currents in response to the rotating electric field of the circularly polarized light. ${ }^{14}$ The latter scales with frequency like $\propto \omega_{0}^{-3}$, thus should be small at optical frequencies. The spin-transfer torques induced by the circular currents might be significant, however. ${ }^{15}$

This perturbation theory is valid in the limit $\delta E_{\text {Stark }}, \varepsilon_{F} \ll$ $E_{g}$, and $\hbar \omega_{0}<E_{g}$. For $n$-GaAs with $E_{g}=1.52 \mathrm{eV}, \Delta=$ $341 \mathrm{meV}, g_{s}^{*} \simeq-0.44, m^{*} \simeq 0.067 m$ and $2 P^{2} / m \simeq 20 \mathrm{eV},{ }^{32}$ a light intensity of $10 \mathrm{GW} / \mathrm{cm}^{2}$ at frequency $\hbar \omega_{0}=1.24 \mathrm{eV}$ $\left(\lambda_{0}=1 \mu \mathrm{m}\right)$ then generates an effective magnetic field of $9 \mathrm{~T}$. This estimate is more than three orders of magnitude larger than what has been predicted in disordered metals involves intraband transitions with the same laser intensities. ${ }^{17,18}$ The high-intensity laser power used in ultrafast optomagnetic experiments leads to heating and demagnetization of samples even at below-band-gap frequencies due to multiple photon absorption, band tails, disorder, etc. The optically induced spin accumulation then can nucleate a persistent magnetization when the samples cools after the pump pulse. ${ }^{4,5}$

\section{B. Ferromagnetic case}

Consider now hole-doped ferromagnetic semiconductors. As before, we assume small hole densities $\varepsilon_{F} \ll E_{g}, \Delta$ and thus limit the discussion to the optical transitions at $\Gamma$. We investigate the weak ferromagnetic regime in which $J \ll \Delta<$ $E_{g}$, therefore it is sufficient to calculate effective fields to the lowest order of $J / \Delta$.

First, we assume an equilibrium magnetization direction $\hat{\mathbf{M}}_{0}$ along the light propagation direction $\hat{\mathbf{q}}$. The average effective field experienced by the valence bands is

$$
\begin{aligned}
\mathbf{B}_{\text {light }}^{(p)} & =\mathbf{B}_{\mathrm{IFE}}^{(p)}+\mathbf{B}_{\mathrm{ICME}}^{(p)} \\
& \simeq-\left(3 \lambda C_{\sigma}^{(p)}+\frac{2 J}{5 \Delta} C_{\rho}^{(p)}\right) \frac{\xi E_{0}^{2} \hat{\mathbf{q}}}{\mu_{B}^{*} g_{s}^{*} \omega_{0}^{2}} .
\end{aligned}
$$


The first term, the helicity $(\lambda)$ dependent term, is the IFE field. The helicity independent term, the second term, corresponds to the ICME field and enhances or suppresses the magnetization even for a linearly polarized beam. The ICME is an odd function of the exchange coupling and, in the smallmagnetization limit, is linearly proportional to the exchange coupling. This is in contrast to the IFE, which is even in the exchange energy and odd in the helicity. In the large-detuning limit, the ICME scales like $\omega_{0}^{-2}$, while the helicity dependent IFE is $\propto \omega_{0}^{-1}$. In this case $\hat{\mathbf{q}} \| \hat{\mathbf{M}}_{0}$, and then the effective field does not trigger magnetization precession dynamics. Linear reversal through zero magnetization can occur if the light-induced effective field is sufficiently larger than the coercive field, which is dramatically reduced down to a few teslas near the Curie temperature. ${ }^{27}$ Magnetization reversal by precession might be possible in principle, but would require much longer light pulses for $\hat{\mathbf{q}} \| \hat{\mathbf{M}}_{0}$.

Second, we consider a magnetization direction perpendicular to the light propagation direction as $\hat{\mathbf{M}}_{0}=\hat{\mathbf{x}} \cos \phi+$ $\hat{\mathbf{y}} \sin \phi$, where $\phi$ is the azimuthal angle. The average lightinduced effective field induced by circularly polarized light on the holes in the valence bands is

$$
\begin{aligned}
\mathbf{B}_{\text {light }}^{(p)} & =\mathbf{B}_{\mathrm{IFE}}^{(p)}+\mathbf{B}_{\mathrm{ICME}}^{(p)} \\
& \simeq-\left(3 \lambda C_{\sigma}^{(p)} \hat{\mathbf{q}}-\frac{J}{5 \Delta} C_{\rho}^{(p)} \hat{\mathbf{M}}_{0}\right) \frac{\xi E_{0}^{2}}{\mu_{B}^{*} g_{s}^{*} \omega_{0}^{2}} .
\end{aligned}
$$

The first term, the IFE field, is along the light wave vector and changes sign with light helicity. The IFE field acts on the holes that relax very fast to generate a spin accumulation $\langle\sigma\rangle_{\mathrm{ne}}^{(p)}$, which by the exchange interactions exerts a strong torque on the local magnetization $\mathbf{T}=J \hat{\mathbf{M}}_{0} \times\langle\boldsymbol{\sigma}\rangle_{\text {ne }}^{(p)} \propto \hat{\mathbf{M}}_{0} \times \mathbf{B}_{\text {light }}^{(p)} \cdot{ }^{33}$ The other term corresponds to the ICME and is strictly longitudinal, which enhances or suppresses magnetization, but does not trigger magnetization precession. ${ }^{34}$ With $J / \Delta \sim 0.1$ we estimate $B_{\mathrm{ICME}}^{(p)} \sim 10^{-4} \mathrm{eV} /\left(\mu_{B}^{*} g_{s}^{*}\right)$ for a light intensity of $10 \mathrm{GW} / \mathrm{cm}^{2}$, which is large considering that $\mu_{B}^{*} g_{s}^{*} \sim$ $10^{-4}-10^{-5} \mathrm{eV} / \mathrm{T}$. Also the effective magnetic field induced by linearly polarized light, in perpendicular configuration $\mathbf{q} \perp \hat{\mathbf{M}}_{0}$, is given by

$$
\begin{aligned}
\mathbf{B}_{\text {light }}^{(p)}= & \mathbf{B}_{\mathrm{ICME}}^{(p)}+\mathbf{B}_{\perp}^{(p)} \\
\simeq & \frac{2 J}{5 \Delta} C_{\rho}^{(p)}\left[\hat{\mathbf{M}}_{0}\left[3 \cos ^{2}(\phi-\alpha)-1\right]\right. \\
& \left.+\frac{3}{2} \hat{\mathbf{q}} \times \hat{\mathbf{M}}_{0} \sin 2(\phi-\alpha)\right] \frac{\xi E_{0}^{2}}{\mu_{B}^{*} g_{s}^{*} \omega_{0}^{2}} .
\end{aligned}
$$

This field has two components, the conventional ICME parallel to $\hat{\mathbf{M}}_{0}$, and a term along $\hat{\mathbf{q}} \times \hat{\mathbf{M}}_{0}$, which exerts a torque on the local magnetization in the $z$ direction. Equations (9) and (10) show that in the perpendicular configuration both linearly and circularly polarized light induce effective fields that exert torques on the equilibrium magnetization and induce precessional dynamics. Note, however, that in our model unpolarized light or just a heat pulse does not generate effective magnetic fields. Linearly polarized light does not carry net angular momentum, but nevertheless induces spin precession by inducing angular momentum transfer between lattice and exchange fields, thereby rotating its plane of polarization

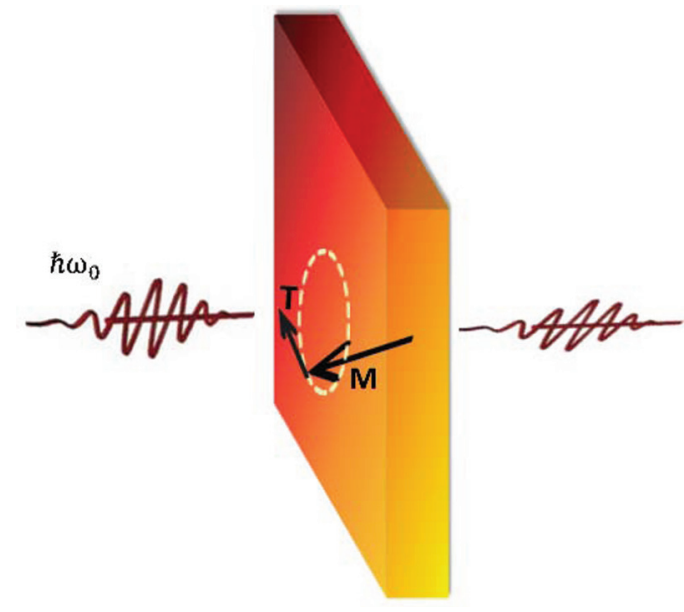

FIG. 2. (Color online) Both linearly and circularly polarized light exert a torque $\mathbf{T}$ on the equilibrium magnetization $\mathbf{M}$, and may trigger magnetization dynamics.

(Faraday effect), see Fig. 2. Circularly polarized photons can directly transfer angular momentum from the light to the spin of electrons only when absorbed. At typical laser intensities, the amount of available angular momentum is by far not enough to reverse the magnetization. We thus demonstrate that in optomagnetism the lattice and exchange fields act as sources and sinks of angular momentum via the spin-orbit and exchange couplings. ${ }^{35}$ We present here a microscopic theory of light-induced magnetic fields. In order to compare with experiments, the magnetization dynamics under effective magnetic field and heat pulses will have to be computed. A realistic micromagnetic simulation in the presence of such an effective field has been carried out in Refs. 5 and 27. A repetition of these calculations for III-V magnetic semiconductors is far beyond the scope of this paper, however.

\section{SUMMARY AND CONCLUSIONS}

In summary, we studied the magnetic response to intense and nonabsorptive, linearly and circularly polarized lights in para- and ferromagnetic III-V semiconductors. The strong spin-orbit coupling plays a vital role to supply the required angular momentum. As a result, the light-induced field strength in GaMnAs is huge, up to several teslas, which is sufficient to reverse magnetization by either linearly or precessional paths. We found that the spin-selective optical Stark effect in ferromagnets induces effective magnetic fields in different directions depending on the light orientation and the magnetization direction.

\section{ACKNOWLEDGMENTS}

This work was supported by EU-ICT-7 Contract No. 257159 "MACALO," the FOM Foundation, the ICC-IMR, and DFG Priority Programme No. 1538 "Spin-Caloric Transport." A.Q. would like to thank A. G. Moghaddam for useful discussions. 
${ }^{1}$ J. Stöhr and H. C. Siegmann, Magnetism: From Fundamentals to Nanoscale Dynamics (Springer-Verlag, Berlin, 2006).

${ }^{2}$ S. Serrano-Guisan, K. Rott, G. Reiss, J. Langer, B. Ocker, and H. W. Schumacher, Phys. Rev. Lett. 101, 087201 (2008),

${ }^{3}$ I. Tudosa, C. Stamm, A. B. Kashuba, F. King, H. C. Siegmann, J. Stöhr, G. Ju, B. Lu, and D. Weller, Nature (London) 428, 831 (2004).

${ }^{4}$ C. D. Stanciu, F. Hansteen, A. V. Kimel, A. Kirilyuk, A. Tsukamoto, A. Itoh, and Th. Rasing, Phys. Rev. Lett. 99, 047601 (2007).

${ }^{5}$ K. Vahaplar, A. M. Kalashnikova, A. V. Kimel, D. Hinzke, U. Nowak, R. Chantrell, A. Tsukamoto, A. Itoh, A. Kirilyuk, and Th. Rasing, Phys. Rev. Lett. 103, 117201 (2009).

${ }^{6}$ A. M. Kalashnikova, A. V. Kimel, R. V. Pisarev, V. N. Gridnev, A. Kirilyuk, and Th. Rasing, Phys. Rev. Lett. 99, 167205 (2007); A. M. Kalashnikova, A. V. Kimel, R. V. Pisarev, V. N. Gridnev, P. A. Usachev, A. Kirilyuk, and Th. Rasing, Phys. Rev. B 78, 104301 (2008).

${ }^{7}$ R. Iida, T. Satoh, T. Shimura, K. Kuroda, B. A. Ivanov, Y. Tokunaga, and Y. Tokura, Phys. Rev. B 84, 064402 (2011).

${ }^{8}$ A. Kirilyuk, A. V. Kimel, and Th. Rasing, Rev. Mod. Phys. 82, 2731 (2010).

${ }^{9}$ P. S. Pershan, J. P. van der Ziel, and L. D. Malmstrom, Phys. Rev. 143, 574 (1966).

${ }^{10}$ Y. R. Shen, The Principles of Nonlinear Optics (Wiley, New York, 1984).

${ }^{11}$ D. Steil, S. Alebrand, A. Hassdenteufel, M. Cinchetti, and M. Aeschlimann, Phys. Rev. B 84, 224408 (2011).

${ }^{12}$ K. Vahaplar, A. M. Kalashnikova, A. V. Kimel, S. Gerlach, D. Hinzke, U. Nowak, R. Chantrell, A. Tsukamoto, A. Itoh, A. Kirilyuk, and Th. Rasing, Phys. Rev. B 85, 104402 (2012).

${ }^{13}$ J. Hohlfeld, C. D. Stanciu, and A. Rebei, Appl. Phys. Lett. 94, 152504 (2009).

${ }^{14}$ B. Talin, V. P. Kaftandjian, and L. Klein, Phys. Rev. A 11, 648 (1975); R. Hertel, J. Magn. Magn. Mater. 303, L1 (2006).

${ }^{15}$ F. K. Joibari and G. E. W. Bauer (unpublished).

${ }^{16}$ S. R. Woodford, Phys. Rev. B 79, 212412 (2009).

${ }^{17}$ V. M. Edelstein, Phys. Rev. Lett. 80, 5766 (1998).

${ }^{18}$ K. Taguchi and G. Tatara, Phys. Rev. B 84, 174433 (2011).

${ }^{19}$ J. A. de Jong, I. Razdolski, A. M. Kalashnikova, R. V. Pisarev, A. M. Balbashov, A. Kirilyuk, Th. Rasing, and A. V. Kimel, Phys. Rev. Lett. 108, 157601 (2012); A. R. Khorsand, M. Savoini, A. Kirilyuk, A. V. Kimel, A. Tsukamoto, A. Itoh, and Th. Rasing, ibid. 108, 127205 (2012); T. A. Ostler, J. Barker, R. F. L. Evans, R. W. Chantrell, U. Atxitia, O. Chubykalo-Fesenko, S. El Moussaoui, L. Le Guyader, E. Mengotti, L. J. Heyderman,
F. Nolting, A. Tsukamoto, A. Itoh, D. Afanasiev, B. A. Ivanov, A. M. Kalashnikova, K. Vahaplar, J. Mentink, A. Kirilyuk, Th. Rasing, and A. V. Kimel, Nat. Commun. 3, 666 (2012).

${ }^{20}$ S. Alebrand, M. Gottwald, M. Hehn, D. Steil, M. Cinchetti, D. Lacour, E. E. Fullerton, M. Aeschlimann, and S. Mangin, Appl. Phys. Lett. 101, 162408 (2012).

${ }^{21}$ M. Y. A. Raja, D. Allen, and W. Sisk, Appl. Phys. Lett. 67, 2123 (1995).

${ }^{22}$ K. S. Burch, D. D. Awschalom, and D. N. Basov, J. Magn. Magn. Mater. 320, 3207 (2008); T. Jungwirth, Jairo Sinova, J. Măsek, J. Kučera, and A. H. MacDonald, Rev. Mod. Phys. 78, 809 (2006).

${ }^{23}$ J. Wang, C. Sun, Y. Hashimoto, J. Kono, G. A. Khodaparast, Ł. Cywiński, L. J. Sham, G. D. Sanders, C. J. Stanton, and H. Munekata, J. Phys.: Condens. Matter. 18, R501 (2006).

${ }^{24}$ N. Tesarova, P. Nemec, E. Rozkotova, J. Subrt, H. Reichlova, D. Butkovicova, F. Trojanek, P. Maly, V. Novak, and T. Jungwirth, Appl. Phys. Lett. 100, 102403 (2012).

${ }^{25}$ C. Phelps, T. Sweeney, R. T. Cox, and H. Wang, Phys. Rev. Lett. 102, 237402 (2009); S. G. Carter, Z. Chen, and S. T. Cundiff, Phys. Rev. B 76, 201308(R) (2007).

${ }^{26}$ M. D. Kapetanakis, I. E. Perakis, K. J. Wickey, C. Piermarocchi, and J. Wang, Phys. Rev. Lett. 103, 047404 (2009); J. Wang, I. Cotoros, D. S. Chemla, X. Liu, J. K. Furdyna, J. Chovan, and I. E. Perakis, Appl. Phys. Lett. 94, 021101 (2009); Y. Hashimoto, S. Kobayashi, and H. Munekata, Phys. Rev. Lett. 100, 067202 (2008).

${ }^{27}$ N. Kazantseva, D. Hinzke, R. W. Chantrell, and U. Nowak, Europhys. Lett. 86, 27006 (2009).

${ }^{28}$ E. O. Kane, J. Phys. Chem. Solids 1, 249 (1957).

${ }^{29}$ G. Abstreiter, M. Cardona, and A. Pinczuk, in Light Scattering in Solids IV, edited by M. Cardona and G. Güntherodt (SpringerVerlag, New York, 1984).

${ }^{30}$ D. Popova, A. Bringer, and S. Blügel, Phys. Rev. B 85, 094419 (2012).

${ }^{31}$ A. H. M. Reid, A. V. Kimel, A. Kirilyuk, J. F. Gregg, and Th. Rasing, Phys. Rev. Lett. 105, 107402 (2010); Phys. Rev. B 81, 104404 (2010).

${ }^{32}$ C. Hermann and C. Weisbuch, Phys. Rev. B 15, 823 (1977).

${ }^{33}$ A. Matos-Abiague and R. L. Rodríguez-Suárez, Phys. Rev. B 80, 094424 (2009).

${ }^{34}$ T. Makino, F. Liu, T. Yamasaki, Y. Kozuka, K. Ueno, A. Tsukazaki, T. Fukumura, Y. Kong, and M. Kawasaki, Phys. Rev. B 86, 064403 (2012).

${ }^{35}$ B. Koopmans, M. van Kampen, J. T. Kohlhepp, and W. J. M. de Jonge, Phys. Rev. Lett. 85, 844 (2000). 